\title{
Study of the Electric Explosion of Titanium Foils in Uranium Salts
}

\author{
Leonid I. Urutskoev ${ }^{1,2}$, Dmitry V. Filippov ${ }^{1}$ \\ ${ }^{1}$ RECOM, National Research Center "Kurchatov Institute", Moscow, Russia \\ ${ }^{2}$ Moscow State University of Printing Arts, Moscow, Russia \\ E-mail: filippov-atom@ya.ru,urleon@ya.ru \\ Received June 9, 2010; revised July 27, 2010; accepted August 2, 2010
}

\begin{abstract}
The results of experiments on electroexplosion titanic foil in water solutions of salts of uranium are presented in this paper. It is shown, that as a result of electroexplosion occurs appreciable (to $20 \%$ ) distortion of an initial isotope parity of uranium. In the most solution parts, observable isotope distortion occurs in favour of enrichment by ${ }^{235} \mathrm{U}$. At the moment of electroexplosion it was not observed an appreciable stream of the neutrons. By means of Cs label and by methods by $\alpha, \beta, \gamma$-spectrometry and mass-spectrometry it have been shown, that isotope distortion occurs at the expense of non-uniform "disappearance" of both isotopes from a solution. The isotope distortion leads to infringement of the ${ }^{234} \mathrm{Th}$ secular equilibrium in the uranyl solution. The equilibrium infringement between the ${ }^{234} \mathrm{Th}$ and ${ }^{234 m} \mathrm{~Pa}$, i.e. within the proper thorium decay chain, was observed also. The assumption about that the effects are caused of low-energy nuclear reactions at the moment of electroexplosion is suggested.
\end{abstract}

Keywords: Electric Explosion, Nuclear Decay

\section{Introduction}

Currently, it is beyond doubt that strong external electromagnetic fields can considerably change the probability of nuclear decay and even change the conditions of nuclear stability [1-7]. For example, complete ionization of ${ }^{187} \mathrm{Re}$ increases the probability of $\beta$-decay (due to decay to a bound electron state) [2], while complete ionization of stable isotopes ${ }^{163} \mathrm{Dy},{ }^{193} \mathrm{Ir},{ }^{205} \mathrm{Tl}$ makes them $\beta^{-}$-active (the half-life time of completely ionized ${ }^{163} \mathrm{Dy}$ was $47 \pm 5$ days [3]). Now it is clear that strong external fields affect to one or another extent the probabilities of all nuclear processes, the major effect being mediated by deformation of the atomic electron states (both occupied by electrons and free) [4-7]. This influence depends appreciably on the decay type and energy, on the degree of prohibition, and, in the general case, on the spins and evenness of the initial and final states of the decaying nucleus. In some cases, the dependence of the change in the decay probability on the intensity of external action can be non-monotonic. Usually, the effect is stronger for hindered (forbidden) decay channels but it is noteworthy that a change in the decay probability induced by exter- nal fields depends on many parameters and every nucleus is in a certain sense unique. For the nuclei that decay along several channels having different parameters and degrees of prohibition, the effect of external fields changes the ratio of decay intensities along different channels. When the fields act on a substance containing a mixture of isotopes, the final ratio of the radioactive isotopes should change as the effects may be different for different isotopes.

It is known that high-current electric explosion of metal wires in a liquid induces strong magnetic fields $(H \sim 1$ MG) and high pulse pressures $\left(P \sim 10^{5} \mathrm{~atm}\right)[8,9]$. To study the effect of external action on the decay of uranium series isotopes, we carried out experiments on the electric explosion of titanium foil in a solution of uranyl sulfate in doubly distilled water. The very idea to investigate impacts of strong electromagnetic fields on the uranium isotopic decay dawned on the authors of this articles resulting from multiple and repeated attempts to grasp the physical mechanism of nuclear reactor runaway which actually took place in the Chernobyl NPP in 1986 [10]. The results of preliminary experiments of this series were published previously [11]. 


\section{Description of the Experimental Setup}

The experiments outlined below were carried out using one of the capacitor banks of the setup described in detail previously [12]. The energy store of the setup was $W \sim$ $22 \mathrm{~kJ}$, the charge voltage was $U=4.8 \mathrm{kV}$, the current amplitude was $I \sim 120 \mathrm{kA}$, and the pulse duration was $\tau$ $\approx 130 \mu$ s. Unlike the explosion chamber used previously [12], the explosion chamber presented in Figure 1 was sealed and allowed mounting of only one electrode. The internal part of explosion chamber 1 was made of polyethylene and was arranged in a lower part of a tough stainless-steel case 2, which ensured air tightness. The voltage was supplied through the upper part of a metal container, which served simultaneously as a gas-collecting chamber 3 with the volume $V \sim 3 \ell$ (prechamber). The gas-collecting chamber is required due to the fact that all attempts to make the gas-water mixture formed during current passage stay in the explosion chamber failed, resulting only in the collapse of the explosion chamber. In a number of experimental series, a prechamber made of organic glass was used to provide the possibility of high-speed optical recording.

Uranium solution 4 with the volume $v \sim 20 \mathrm{~cm}^{3}$ was poured into the internal part of explosion chamber 1. Two types of solutions were used, namely, a solution of uranyl sulfate enriched in ${ }^{235} \mathrm{U}$ and a solution of uranyl nitrate with the natural isotope distribution of uranium. The concentration of the uranium solutions used in experiments varied from $10^{-2}$ to $10^{-1} \mathrm{~g}$.-mol/liter. A bulk central titanium electrode 5 bearing foil 6 welded to it

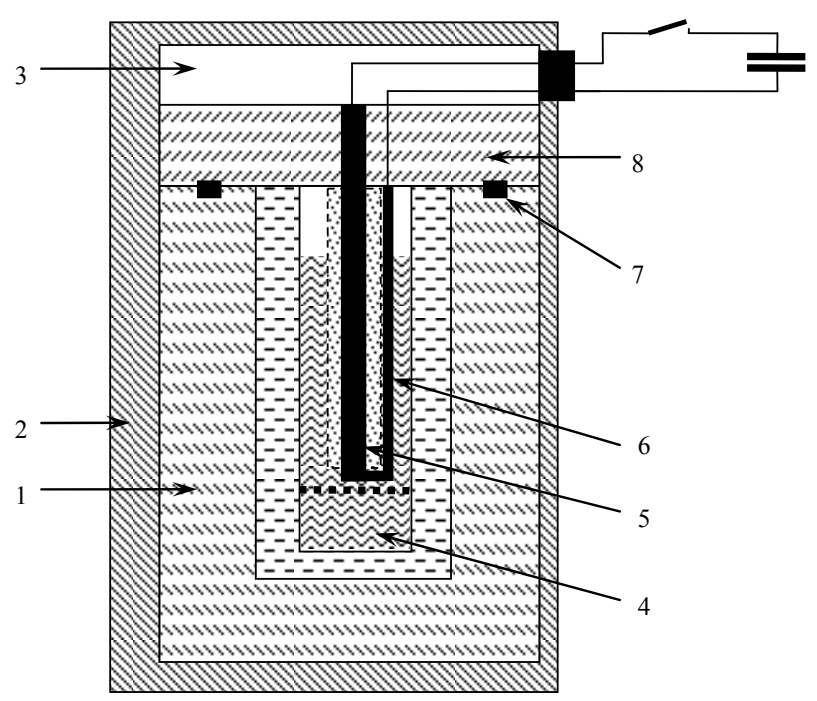

Figure 1. Schematic drawing of experimental setup: 1-interior of the explosion chamber; 2-stainless steel frame; 3-gas-collecting chamber; 4-liquid; 5-titanium electrode; 6-titanium foil; 7-tightening; 8-lid. was immersed in the solution. Thus, the welded foil acted as a cable armoring short-circuited to the cable central core. The amount of the titanium foil (load) varied in different experiments from one to four $1 \mathrm{~cm}$-wide bands, each with the thickness $\Delta=50 \mu \mathrm{m}$ and the length $L=4.0 \mathrm{~cm}$. The weight of each band was $m=(90 \pm 5) \times$ $10^{-3} \mathrm{~g}$.

The discharge parameters were monitored using coaxial shunts and a voltage-ratio box to record current and voltage pulses. The signals were recorded by means of an AD converter with a sampling rate of $1 \mathrm{GHz}$. Typical current and voltage oscillograms were presented in our earlier publications $[12,13]$.

It is well known that electric explosion of a conductor in water produces an intense electromagnetic radiation $[8,9]$, which requires taking special measures to provide the interference immunity of the signals. Note that if an uranium solution is used as the liquid in electric explosion, the level of electromagnetic radiation increases by a large factor, especially in the second half of the pulse duration.

During current passage, $\gamma$-radiation was recorded. This was done by scintillator-based detectors (NaI, CsI) and photomultipliers, which were placed 1.5 to 2 meters away from the location of electric explosion. No noticeable excess over the background $\gamma$-radiation during the current pulse was detected.

The neutron yield was also monitored. For sufficiently efficient recording of fission neutrons, a special neutron detector was assembled from nine slow neutron counters (CHM-17). The whole assembly was surrounded by a 8 $\mathrm{cm}$-thick polyethylene moderator. The electromagnetic interference immunity was provided by placing the whole detector block together with preamplifiers inside a copper screen. For elimination of false pulses (common-mode interferences), the slow neutron counters were split into two groups.

\section{The Procedure of Measurements}

For determining the efficiency of neutron detection, a neutron calibration source ${ }^{252} \mathrm{Cf}$ was placed in the explosion chamber. The measured detection efficiency of fission neutrons was $0.04 \%$. Every detected pulse was identified as a neutron if it coincided with the pulse from the calibration source in the shape and amplitude. It follows from the measurements that the neutron flux after electric explosion did not exceed $I<10^{3}$ neutrons per pulse.

Note that in the experiments using organic glass prechamber 3, the neutron detector also recorded some pulses other than neutron pulses. Figure 2(a) shows a neutron signal from the calibration source and a typical "false" signal. It follows from Figure 2 that duration of 


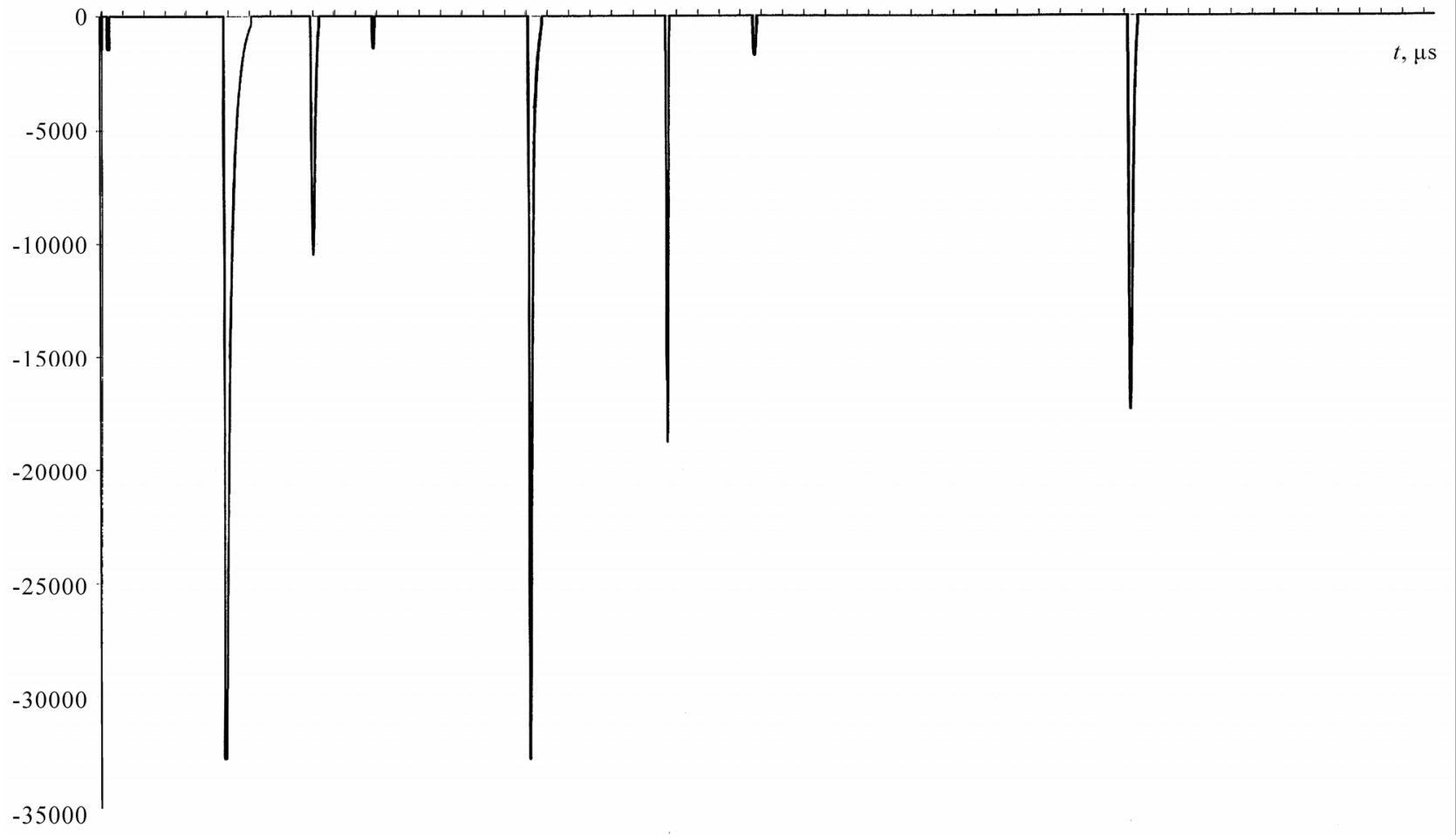

(a)

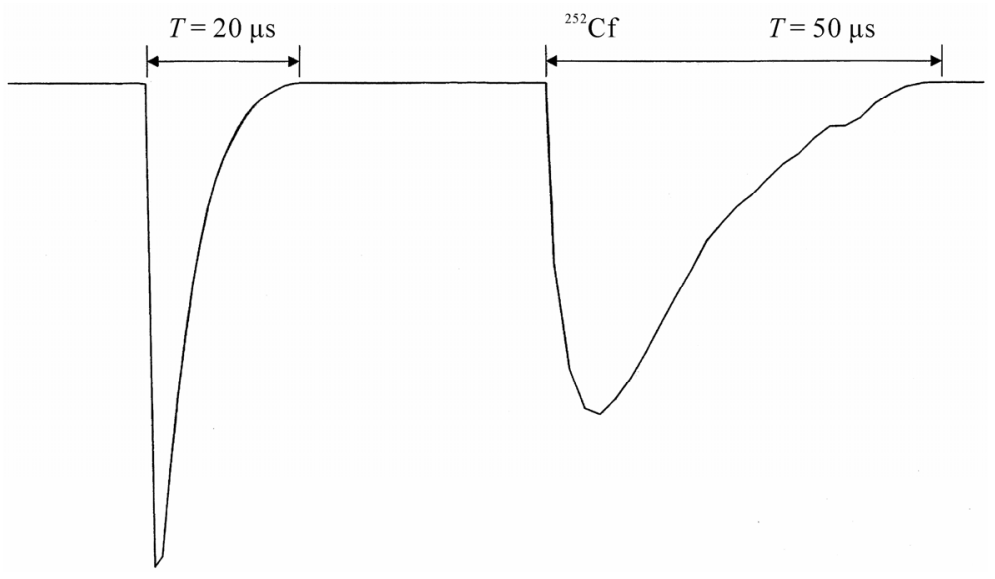

(b)

Figure 2. Signals of the neutron detector: (a) a typical distribution of the signals in time; (b) a "false" pulse and the pulse from the calibration source.

the "false" pulse differs by a factor of about 2 from the neutron pulse duration. This difference is manifested in both electronic and ionic components of the signal. The number of detected "false" pulses was much greater than the number of neutron pulses. A typical distribution over the times of signal arrival to the detector is shown in Figure 2(b). As regards the shape, the "false" signals resemble $\gamma$-quanta, which were also detected by the neutron detector, although with a lower efficiency. However, these signals could not be identified as $\gamma$-quanta as in this case, they would have been detected much more efficiently by $\gamma$-radiation detectors. These signals could not be attributed to the electromagnetic pickup either, because they were detected after operation of the capacitor battery. The nature of the "false" signals remained obscure but there is more evidence for their electromagnetic origin than for nuclear origin.

Upon application of current pulses on a titanium foil, 
the foil explodes and the pressure in the explosion chamber sharply increases. For this reason, most of the uranium solution breaks to prechamber 3 through seal 7 . This gives two different solution volumes, and, according to preliminary results [11], they differed substantially in the isotope composition of uranium. For this reason, sampling of the uranium solution after the "shot" was performed from both upper (hereinafter "up") and lower (hereinafter "lw") volume. For convenience of collecting the solution, special groove was envisaged in the polyethylene lid 8.

The samples were taken by a disposable syringe, placed in standard $2-\mathrm{m} \ell$ or $5-\mathrm{m} \ell$ plastic tubes and immediately sealed by PARAFILM "M". The dosage accuracy was relatively low being equal to $\sim 5 \%$. Therefore, the measured volumetric activity values were used only for rough estimates and the results of $\gamma, \alpha$, and $\beta$ spectrometry measurements were represented as ratios of the values obtained in one and the same measurement. This presentation of measurement results ensured minimization of possible errors caused by dosage errors.

In order to avoid procedural errors and to enable comparison of the results of measurements of the same values obtained by different procedures, two samples with identical volumes were measured for each procedure. One sample (reference sample, "rs") contained the initial solution of uranium for this experiment and the other sample (experimental sample, "ex") was the uranium solution taken from the explosion chamber after the electric explosion. This made it possible to compare the results of, for example, $\gamma$ - and mass spectrometry for experiments carried out with natural and isotope-enriched solutions of uranium by plotting the results together as the ratio $R=\left({ }^{235} \mathrm{U} /{ }^{238} \mathrm{U}\right)_{\mathrm{ex}} /\left({ }^{235} \mathrm{U} /{ }^{238} \mathrm{U}\right)_{\mathrm{rs}}$.

Laser mass spectrometry was chosen as the procedure for direct determination of the elemental and isotope compositions. Having rather high accuracy $\left(10^{-4}-10^{-5}\right.$ at $\%$ ), this procedure gave an error of determination of trace impurities of about $10-15 \%$. The accuracy of determination of the isotope composition of elements was $1.5-2 \%$ for minor isotopes and less than $1 \%$ for principal isotopes, which is consistent with published data [14]. During our study, the samples taken from all "shots" were measured by mass spectrometry but the most thorough isotope analysis was carried out for the following chemical elements: Fe, Ti and U. The results of measurements for initial components and samples from one experiment with a solution of enriched uranyl sulfate are summarized in Table 1. The errors of measurements of percentages of all chemical elements and isotopes presented in Table 1 fall into the corridor of errors discussed above.

The independent measurements of the uranium isotope
Table 1. Elemental and isotope compositions of the samples, $\%$.

\begin{tabular}{|c|c|c|c|}
\hline & Control & Contr. foil & Exp \\
\hline $\mathrm{Na}$ & 0.135 & 0.0000 & 4.380 \\
\hline $\mathrm{Mg}$ & 0.001 & 0.0056 & 0.170 \\
\hline $\mathrm{Al}$ & 0.060 & 0.0032 & 0.550 \\
\hline $\mathrm{Si}$ & 0.117 & 0.0067 & 0.500 \\
\hline $\mathrm{P}$ & 0.037 & 0.0025 & 0.015 \\
\hline S & 49.250 & 0.0038 & 3.750 \\
\hline $\mathrm{Cl}$ & 0.321 & 0.0044 & 0.093 \\
\hline K & 0.047 & 0.0050 & 3.500 \\
\hline $\mathrm{Ca}$ & 0.034 & 0.0163 & 1.970 \\
\hline $\mathrm{Ti}$ & 0.434 & 99.8606 & 80.080 \\
\hline V & 0.002 & 0.0018 & 0.002 \\
\hline $\mathrm{Cr}$ & 0.002 & 0.0121 & 0.039 \\
\hline $\mathrm{Mn}$ & 0.005 & 0.0020 & 0.023 \\
\hline $\mathrm{Fe}$ & 0.077 & 0.0694 & 0.690 \\
\hline $\mathrm{Ni}$ & 0.009 & 0.0060 & 0.013 \\
\hline $\mathrm{Co}$ & 0.002 & 0.0002 & 0.009 \\
\hline $\mathrm{Cu}$ & 0.005 & 0.0002 & 0.021 \\
\hline $\mathrm{Zn}$ & 0.011 & 0.0000 & 0.045 \\
\hline $\mathrm{Zr}$ & 0.000 & 0.0003 & 0.000 \\
\hline $\mathrm{Ag}$ & 0.000 & 0.0000 & 0.510 \\
\hline U & 49.453 & 0.0000 & 3.610 \\
\hline${ }^{235} \mathrm{U}$ & 21 & & 24.56 \\
\hline${ }^{238} \mathrm{U}$ & 79 & & 75.44 \\
\hline${ }^{46} \mathrm{Ti}$ & 8.0 & & 8.00 \\
\hline${ }^{47} \mathrm{Ti}$ & 7.3 & & 8.07 \\
\hline${ }^{48} \mathrm{Ti}$ & 73.8 & & 71.48 \\
\hline${ }^{49} \mathrm{Ti}$ & 5.5 & & 6.16 \\
\hline${ }^{50} \mathrm{Ti}$ & 5.4 & & 6.27 \\
\hline
\end{tabular}

ratio and concentrations of some of its decay products were accomplished using liquid scintillation $\alpha, \beta$-spectrometry and solid-state $\alpha$-spectrometry procedures.

The counting sample for liquid scintillation spectrometry was prepared in the following way. A $10-\mu \ell$ 
aliquot portion of the test solution was thoroughly mixed with the scintillating solution ULTIMA GOLD AB. The counting sample obtained in this way was measured by a TRI-CARB 2550 TR/AB counter (Canberra-Packard) equipped with a time discriminator of alpha and beta pulses and a multichannel analyzer. The identification and activity calculation of isotopes were performed using a special program Spectradec for spectrum processing, which makes use of the library of instrumental spectra of separate isotopes. The result was found by comparing the studied convoluted instrumental spectrum with this spectrum obtained by simulation. This procedure has substantial errors in determination of most isotopes and, hence, this analysis was semiquantitative.

The contents of alpha-emitting isotopes were determined on a standard Canberra $\alpha$-spectrometric complex, which comprised four $\alpha$-spectrometers of design 7401 with PIPS detectors. The active area of each detector was $600 \mathrm{~mm}^{2}$. The counting sample was prepared by electrochemical deposition of the emitters from a solution of sodium ammonium sulfate at $\mathrm{pH} \sim 2.2-2.5$ onto stainlesssteel polished discs.

To decrease the effect of conversion electrons, the measurements were carried out at a $14 \mathrm{~mm}$ distance from the detector surface. This effect was manifested as asymmetry of the right "shoulder" of the peaks of the instrumental $\alpha$-spectrum, giving rise to an additional error in their approximation. The average time of measurement was $\sim 1.5 \times 10^{5} \mathrm{~s}$. The quality of the prepared counting sources was estimated based on ${ }^{234} \mathrm{U}$ peak resolution at the $4774.6 \mathrm{keV}$ line (yield $72.4 \%$ ). The energy resolution of the measurements was at least 23.3 $\mathrm{keV}$, while the contribution of the ${ }^{234} \mathrm{U}$ radiation peak base to the low-energy ${ }^{235} \mathrm{U}$ and ${ }^{238} \mathrm{U}$ isotopes was less than $0.1 \%$ and could be properly taken into account.

The isotope ratios were calculated from the peak areas. Since ${ }^{235} \mathrm{U}$ gives rise to seven intense lines which overlap with all $\alpha$-decaying nuclei present in the mixture $\left({ }^{234} \mathrm{U}\right.$, $\left.{ }^{236} \mathrm{U},{ }^{238} \mathrm{U}\right)$, this was calculated using the algorithm implemented in RadSpectraDee software. This implies approximation of peaks by an intricate function combining an asymmetric Gaussian, an exponent and a hyperbola.

In the $\gamma$-spectrometric procedure, a $40 \mathrm{~cm}^{3}$ germanium detector and a standard Canberra spectrometer were used. The energy resolution of the gamma-spectrometer determined from the ${ }^{137} \mathrm{Cs}$ line $(662 \mathrm{keV})$ was $1.8 \mathrm{keV}$. The effect of geometric factor on the results was eliminated by mounting samples right on the detector end face using a specially manufactured holder. Since the fine titanium powder suspended in an uranium solution precipitated over 24 hours and Th was found to interact with titanium oxides, then in order to avoid additional errors in the measurements, the uranium solution container was mounted in a strictly horizontal position relative to the ground surface. In order to eliminate the possible errors related to the time drift of $\gamma$-spectrometer parameters, the experimental and reference samples of uranium solution were measured in turn.

The spectra were processed in the energy regions of 92 $\mathrm{keV}\left(92.38 \mathrm{keV}\right.$ and $92.8 \mathrm{keV}$ are $\gamma$-lines of ${ }^{234} \mathrm{Th}$, which is a daughter product of $\left.{ }^{238} \mathrm{U}\right), 1 \mathrm{MeV}(1001 \mathrm{keV}$ is the $\gamma$-line of ${ }^{234 m} \mathrm{~Pa}$, a daughter product of $\left.{ }^{234} \mathrm{Th}\right)$ and $186 \mathrm{keV}$ ( $185.7 \mathrm{keV}$ is the $\gamma$-line of ${ }^{231} \mathrm{Th}$, a daughter product of $\left.{ }^{235} \mathrm{U}\right)$. Single peaks were first approximated by a Gaussian and then the areas under them were calculated. The region of $\gamma$-doublet at $92.5 \mathrm{keV}$ shows an overlap of the ${ }^{234} \mathrm{Th}$ and ${ }^{231} \mathrm{~Pa} \gamma$-lines $(93.063 \mathrm{keV})$ with the $\mathrm{K}_{\alpha} \mathrm{X}$-ray lines of actinides, resulting in a complex background. The uranium $\mathrm{K}_{\alpha 1}$ and $\mathrm{K}_{\alpha 2} \mathrm{X}$-ray lines appear due to uranium self-fluorescence, the $\mathrm{K}_{\alpha 2}$ peak contributing directly to the $92.5 \mathrm{keV}$ doublet. The contribution of thorium $\mathrm{K}_{\alpha 1}$ and $\mathrm{K}_{\alpha 2}$ lines related to ${ }^{235} \mathrm{U}$ decay was negligibly small. The resolution of the spectrum at 82-102 keV into components was done by a computer program.

\section{Experimental Results}

As shown by preliminary studies [11], an electric explosion of titanium foil in a solution of uranyl sulfate induces a distortion of the initial $U$ isotope ratio and disturbance of the ${ }^{234} \mathrm{Th}$ secular equilibrium. Our study confirmed the conclusions of the preliminary experiments and, resorting to additional procedures, provided a more detailed picture of the phenomenon. The use of both natural and enriched $U$ in the experiments increased the accuracy of measurements and provided a more reliable knowledge of the process. For instance, experiments with enriched $U$ allowed us to attain a satisfactory accuracy in determining the uranium isotope ratio by mass spectrometry, and the use of natural uranium markedly increased the accuracy of measurement of the uranium isotope ratio from the ratio of 186/1001 $\gamma$-lines.

When using different procedures we often faced the so-called "first measurement problem". In liquid $\beta$ spectrometry and solid-state $\alpha$-spectrometry, this was a distortion of the studied spectrum. In $\beta$-spectrometry, the effect was manifested as an increase in the fraction of conversion electrons, and in $\alpha$-spectrometry, this was appearance of an additional low-energy $\alpha$ peak. In the measurements by $\gamma$-spectrometric procedure, the "first measurement effect" was in the fact that the measurement that was performed first differed most often from the subsequent measurements by a value markedly exceeding three standard deviations. After several hours, even in the second measurement, the effect usually dis- 
appeared for all procedures.

As reported in a previous study [11], the distortion of the uranium isotope ratio from mass spectrometry data averaged over nine runs was $R^{\text {up }}=1.18 \pm 0.07$ for the upper sample and $R^{\mathrm{lw}}=0.94 \pm 0.01$ for the lower sample. These results could seemingly be interpreted as a usual separation of $U$ isotopes but the addition of ${ }^{137} \mathrm{Cs}$ radioactive label to the initial solution ruled out the possibility of this interpretation. Figure 3 shows the results of measurements of the upper samples $\left(R^{\text {up }}\right)$ obtained by different procedures. The data were averaged over a series of 15 runs. It can be seen from the Figure that the results of measurements by all three procedures are in satisfactory agreement with one another.

The two rightmost points correspond to $\gamma$-spectrometric measurement of the concentration of $U$ isotopes with respect to the cesium label. Thus, it became clear that uranium enrichment is caused by the fact that the concentration of both $\mathrm{U}$ isotopes in the solution decreases at the "shot" instant as a result of some process. Moreover, the ${ }^{238} \mathrm{U}$ concentration decreases more appreciably than the ${ }^{235} \mathrm{U}$ concentration, and this is perceived as "effective" enrichment. The same conclusion can be reached relying on the data obtained by $\alpha$-spectrometric procedure where an almost twofold decrease in the ${ }^{238} \mathrm{U}$ specific volume activity was detected in some experiments. The ${ }^{235} \mathrm{U}$ depletion effect noted for the lower sample [11] was less pronounced than the enrichment effect of the upper sample, $R^{\text {lw }}<R^{\text {up }}$, and could become totally unobservable upon a change in the experimental conditions. For example, upon the addition of heavy water to a solution of uranyl sulfate $\left(\mathrm{UO}_{2} \mathrm{SO}_{4}+10 \% \mathrm{D}_{2} \mathrm{O}\right)$, the ${ }^{235} \mathrm{U}$ depletion effect in the lower sample disappeared to within the experimental errors, while $R^{\text {up }}$ decreased insignificantly and remained $R^{\text {up }}>1$.

The second macroscopically significant effect noted in [11] was the disturbance of ${ }^{234} \mathrm{Th}$ secular equilibrium observed in both upper and lower samples almost in all experiments. However, most often, the disturbance of ${ }^{234} \mathrm{Th}$ equilibrium was more pronounced in lower samples. Figure 4 shows a typical result of $\gamma$-measurements for the upper sample from run No.975 illustrating the disturbance of the ${ }^{234} \mathrm{Th}$ secular equilibrium. The concentration of uranium sulfate solution in this run was $C=$ $10^{-1} \mathrm{~mol} / \ell$. The result is presented as the time dependence $R_{\gamma}^{\text {up }}(t)$ plotted as the intensity ratio of the ${ }^{231} \mathrm{Th}$ $186 \mathrm{keV}$ line (this line can be regarded as having no time dependence) to the $92 \mathrm{keV}$ line of ${ }^{234} \mathrm{Th}$, which is in secular equilibrium with ${ }^{238} \mathrm{U}$. The value $t=0$ corresponds to the "shot" instant. Figure 4 shows the time course from $T \sim 600 \mathrm{~h}$, which approximately corresponds to ${ }^{234} \mathrm{Th}$ and ${ }^{234 m} \mathrm{~Pa}$ accumulation periods.

Figure 5 shows the results of time $\gamma$-measurements of the upper sample for run No.1037 in which a solution of uranyl nitrate $\mathrm{UO}_{2}\left(\mathrm{NO}_{3}\right)_{2}$ with the initial natural uranium isotope distribution was used. The time dependence of the enrichment factor is represented as the ratio of two pairs of $\gamma$-lines, $R_{186 / 92}^{\text {up }}$ and $R_{186 / 1001}^{\text {up }}$. It can be seen from Figure 5 that the equilibrium between the $92 \mathrm{keV}$ and $1001 \mathrm{keV} \gamma$-lines was disturbed to a statistically significant extent only in the second measurement (the "first measurement" problem) and the disturbance was retained for approximately $500 \mathrm{~h}$ (approximately the ${ }^{234} \mathrm{Th}$ accumulation period). Then the intensity of both $\gamma$-lines started to decrease with respect to the reference sample,

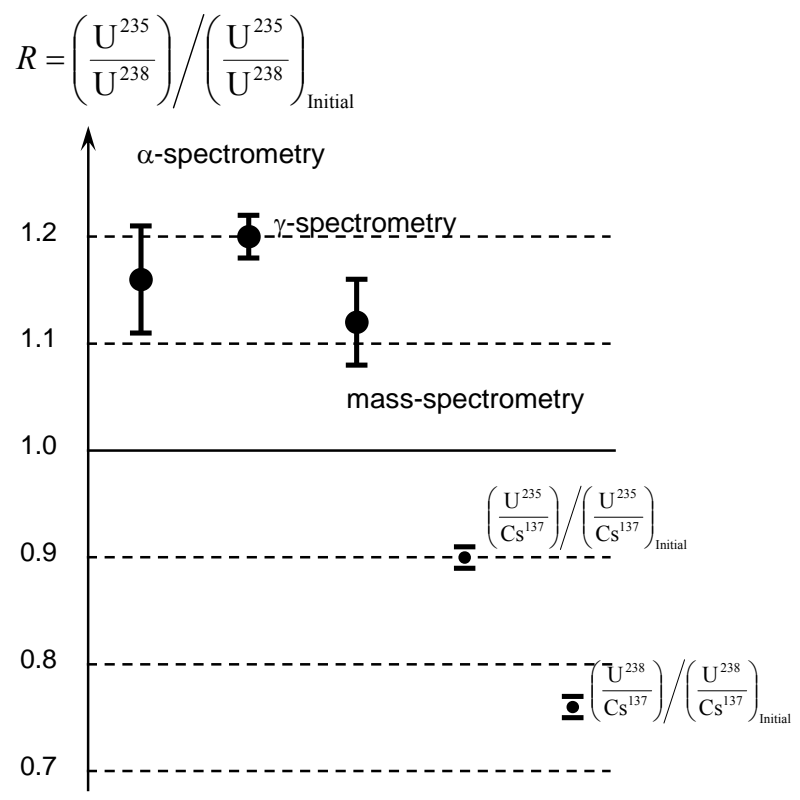

Figure 3. Results of measurements of the "upper" samples $\left(R^{\text {up }}\right)$ obtained by different procedures.

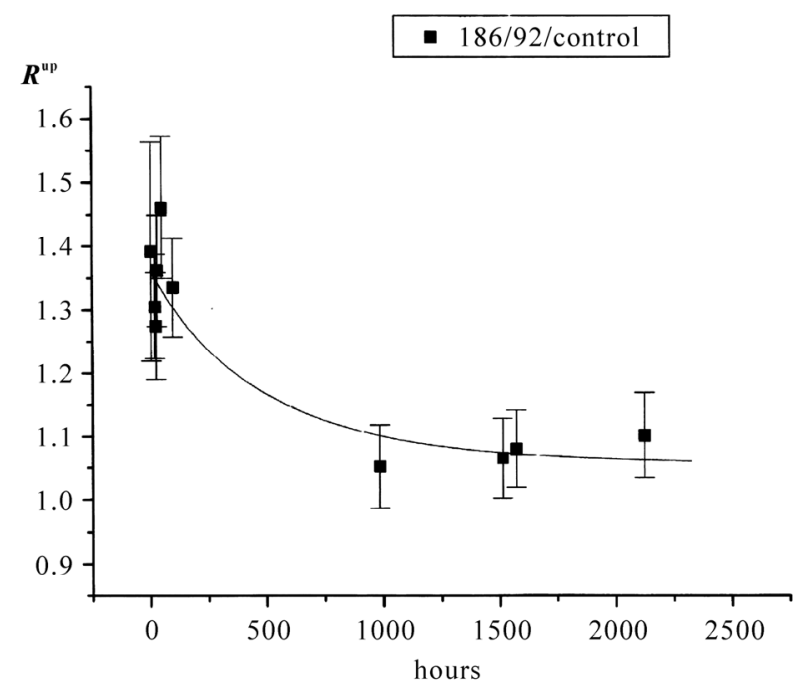

Figure 4. Typical result of $\gamma$-measurements of $R^{\text {up }}=\left(I^{186} /\right.$ $\left.I^{92.5}\right)_{e x} /\left(I^{186} / I^{92.5}\right)_{\text {rs }}$ (the sample of run No. 975). 


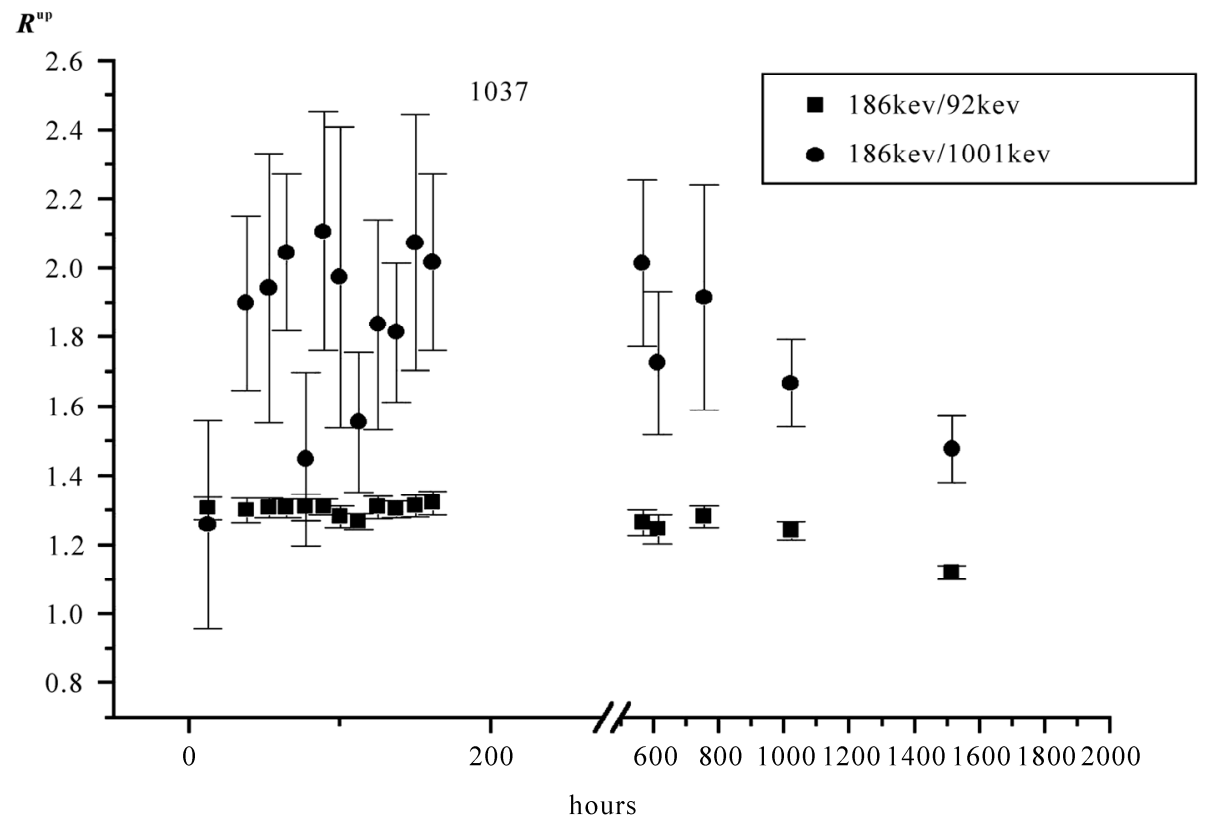

Figure 5. The same ratio as at Figure $4, R^{\mathrm{up}}=\left(I^{186} / \mathbf{I}^{92.5}\right)_{\mathrm{ex}} /\left(I^{186} / I^{92.5}\right)_{\mathrm{rs}}$ and $R^{\mathrm{up}}=\left(I^{186} / \mathbf{I}^{1001}\right)_{\mathrm{ex}} /\left(I^{186} / I^{1001}\right)_{\mathrm{rs}}$ for run No. 1037 .

and by approximately the $5000 \mathrm{~h}$ after the "shot", the equilibrium was restored. The question of whether different chemical compositions of the uranyl solution or the difference in the initial isotope distribution is responsible for this qualitatively different behaviors remained open.

\section{Conclusions}

The key experimental results presented in this paper can be summarized as follows.

1) The electric explosion of a titanium foil in an uranyl salt entailed a marked distortion of the initial $U$ isotope distribution in the solution. The "lower" sample $(v \sim 2-3$ $\left.\mathrm{cm}^{3}\right)$ shows depletion in ${ }^{235} \mathrm{U}\left(\mathrm{R}^{1 \mathrm{w}}=0.94 \pm 0.01\right)$, while the "upper" sample $\left(v \sim 10 \mathrm{~cm}^{3}\right)$ shows a more pronounced enrichment $\left(\mathrm{R}^{\text {up }}=1.18 \pm 0.07\right)$.

2) The processes initiated by the electric explosion result in a decrease in the specific concentrations of both $U$ isotopes but the ${ }^{238} \mathrm{U}$ concentration decreases to a larger extent, giving rise to "enrichment effect".

3) At the instant of electric explosion, no induced uranium fission is observed and no fission neutrons are detected.

4) Within 1-3 ms after the end of current pulse, gas counters filled with ${ }^{3} \mathrm{He}$ detected some signals having, in all probability, electromagnetic origin.

5) At the instant of electric explosion, the ${ }^{234} \mathrm{Th}$ secular equilibrium in the uranyl solution was disturbed. The most pronounced disturbance of the secular equilibrium was observed in "lower" samples, and subsequently the equilibrium was restored with the period $T=24.5$ days. In the "upper" samples, the ${ }^{234} \mathrm{Th}$ equilibrium was disturbed to a much lesser extent and the time variation was almost missing.

6) In some experiments, $\gamma$-measurements of the "upper" samples revealed disturbance of the equilibrium between the ${ }^{234} \mathrm{Th} 92.5 \mathrm{keV}$ doublet and the $1001 \mathrm{keV}$ $\gamma$-line of its daughter product, ${ }^{234 m} \mathrm{~Pa}$, i.e. within the proper thorium decay chain.

The electric explosion of a titanium foil in a liquid produces a considerable amount of gases by different mechanisms (pyrolytic decomposition of water, titanium oxidation and so on). Thus in the experiments in question, the pressure in the prechamber $(V \sim 3 \ell)$ increased after the electric explosion of the foil by $\Delta P \sim 1$ atm. This should result in an increase in the specific volume activity of all radioactive isotopes present in the initial solution. Indeed, the results of measurements carried out by different procedures ( $\alpha, \beta, \gamma$-spectrometry) indicate that this effect was actually observed in some cases.

However, in most experiments, an unequal decrease in the specific activity was found for ${ }^{238} \mathrm{U}$ and ${ }^{235} \mathrm{U}$ isotopes. As noted above, no effect of induced uranium fission was observed and, hence, this effect cannot be responsible for the decrease in the specific activity and for the distortion of the initial uranium isotope ratio.

\section{Discussion}

On the basis of available experimental data, the following interpretation appears most likely. The key processes 
resulting in the "effective" uranium enrichment take place in the area of current passage and in the area directly adjoining the plasma channel, since the major energy contribution is made in this area and strong magnetic fields and high pressures develop at the pulse instant. As regards the space marked by a dashed line in Figure 1, only a pressure pulse is transmitted there, which is apparently insufficient to give rise to the "enrichment" effect. The amount of the solution remaining on the bottom of the explosion chamber after the electric explosion was in line with this interpretation.

This fact could possibly be explained by assuming that the decrease in the specific radioactivity of uranium and other radioactive isotopes is a consequence of low-energy nuclear reactions. Below we call this hypothetical phenomenon the low energy transformation of nuclei (hereinafter LET [15]). Indeed, since the electric explosion of a titanium foil in both distilled water $[12,16,17]$ and uranium salt resulted in depletion of the natural titanium isotope mixture in ${ }^{48} \mathrm{Ti}$ isotope, it is reasonable to assume that this phenomenon is caused by the same physical mechanism in both cases. The experimental research of the regular features of LET that we have carried out for many years showed that even-even isotopes of chemical elements with atomic numbers divisible by $\times 16(A=16$ is oxygen) are more prone to be transformed than their neighbors.

Yet another hypothetical mechanism responsible for the uranium isotope distortion could be a change in $\alpha$-decay periods caused by either ionization or action of intense magnetic fields. The atomic electrons increase the $\alpha$-decay probability with respect to that for a completely ionized atom. First, the field of atomic electrons decreases the barrier for an $\alpha$-particle and, second, the nuclear charge decreases by 2 units upon $\alpha$-decay, which changes the electron shell energy. Taking account of the effect of atomic electrons results in the necessity of replacing energy of an $\alpha$-particle in the calculation of the $\alpha$-decay constant by an "effective" energy, which is greater than the real energy $E \rightarrow E+73 Z^{4 / 3}+65 Z^{5 / 3} \mathrm{eV}$ [18]. Due to the exponential dependence of the $\alpha$-decay probability on the $\alpha$-particle energy $\ln p \propto$ const $-\sqrt{E}^{-1}$, the effect of atomic electrons may be pronounced. As was to be expected, the atomic electrons affect low-energy processes to a larger extent. For example, for ${ }^{147} \mathrm{Sm}$ ( $\alpha$-particle energy $\sim 2.31 \mathrm{MeV} ; T_{1 / 2}=7 \times 10^{11}$ years), the presence of the electron shell increases the probability of $\alpha$-decay 2.6 -fold compared with the nucleus of a fully ionized atom [18].

The influence of a superstrong magnetic field on the $\alpha$-decay probability can be qualitatively described in the following way. An external superstrong magnetic field changes the energy of the atomic electron shell [19] and, hence, changes also the energy of any nuclear decay, as the decay energy is equal to the difference between the total energies of the initial and final systems with allowance for ionization energies of atoms or ions [1]. For $\alpha$-decay, the presence of an external superstrong magnetic field results in an increase in the decay energy and, hence, in an increase in the $\alpha$-decay probability. Since the ${ }^{238} \mathrm{U} \alpha$-decay energy is lower than the energies of the principal channels of ${ }^{235} \mathrm{U} \alpha$-decay, the relative increase in the ${ }^{238} U \alpha$-decay probability caused by ionization and by the effect of an external superstrong magnetic field on the atomic electron shell would be more pronounced than that the relative increase in the ${ }^{235} \mathrm{U} \alpha$-decay probability. On the other hand, an external magnetic field changes the geometry of the problem: the spherical symmetry is replaced by the preferential direction along the magnetic field. This effect is ambiguous and would be considerably different for the even-even ${ }^{238} \mathrm{U}$ nucleus having a zero magnetic moment and the ${ }^{235} \mathrm{U}$ nucleus where the $\alpha$-decay occurs between states of nuclei with nonzero spins.

It can be seen without difficulty that since the abovediscussed effects have different signs with respect to the change in the specific volume activity of isotopes, then depending on the experimental conditions, either an increase or a decrease in the resulting specific activity with respect to different $\mathrm{U}$ isotopes is possible. This is actually observed in experiments.

One more reason supporting LET is the disturbance of ${ }^{234} \mathrm{Th}$ secular equilibrium observed in experiments. The disturbance of the equilibrium was due to a decrease in the ${ }^{234} \mathrm{Th}$ specific concentration in an uranyl solution. This conclusion follows from data of $\beta$ and $\gamma$-spectrometry. Indeed, if only the ${ }^{238} \mathrm{U}$ specific concentration decreased at the instant of electric explosion, then the specific $\beta$-activity of ${ }^{234} \mathrm{Th}$ would decrease with the pe$\operatorname{riod} T=24.5$ days. However, the sharp drop of the specific $\beta$-activity was detected during the first 24 hours after the electric explosion. The same conclusion can be drawn from the $\gamma$-intensity ratio of $E=92.5 \mathrm{keV}$ lines of the sample and the initial solution, which was $I_{\mathrm{ex}}^{92.5} / I_{\mathrm{rs}}^{92.5}<1$. Thus, the sign of this effect and the fact of the subsequent ${ }^{234} \mathrm{Th}$ accumulation with time preclude interpreting the disturbance of secular equilibrium as being due to the decrease in the ${ }^{238} \mathrm{U}$ concentration alone.

As has already been noted in the Introduction, the macroscopic nature of the observed distortions of the initial uranium isotope ratio implies that the phenomenon responsible for this effect occurs in a solution volume considerably exceeding the plasma channel volume. This is evidence supporting the hypothesis that some radiation arises during the electric explosion [12]. The detection of pulses of obviously electromagnetic origin by gas detec- 
tors is indirect evidence in favor of the electromagnetic nature of the arising radiation.

It is noteworthy that very similar signals but in much lower quantities were also detected in those experiments with the organic-glass prechamber in which $\mathrm{H}_{2} \mathrm{O}, \mathrm{D}_{2} \mathrm{O}$ or aqueous glycerol were used instead of the uranyl solution. Several recent publications reported the observation of neutrons in high-voltage electrolysis in $\mathrm{D}_{2} \mathrm{O}$ or during cavitation (e.g., [20]). In those cases where the neutron spectrum was not directly measured and neutron detectors were used in the counting mode, an error could arise in the interpretation of the results of measurements due to detection of "false" pulses.

The disturbance of the equilibrium between ${ }^{234} \mathrm{Th}$ and its daughter ${ }^{234 m} \mathrm{~Pa}$ nucleus detected by $\gamma$-spectrometry can be a result of the change in the $\beta^{-}$-decay probabilities along different channels. This item was discussed in more detail previously [21].

Note that the specific features arising during the spectrometric measurements and observed during the first 24 hour after electric explosion (the "first measurement" problem) coincide, as regards the time scale of the effect, with the period for disappearance of the deformation of ${ }^{57} \mathrm{Fe}$ Mössbauer spectrum noted previously [13]. Coincidence of the time scales of these effects can suggest a common origin.

Thus, summarizing the results of these studies provides the following conclusions. Additional evidence supporting the emergence of lepton type "strange" radiation at the instant of electric explosion of a conductor in a liquid was obtained [12]. The electric explosion of titanium foil in an uranium salt was shown to initiate a number of phenomena with still obscure physical mechanisms. It can be seen from the presented experimental results that all the observed effects are closely related to one another but the available experimental data do not allow their final separation into constituents.

\section{Acknowledgements}

The authors wish to express their gratitude to staff members of the Russian Scientific Center Kurchatov Institute V. L. Kuznetsov and S. V. Zhukov for performing numerous measurements. We are grateful to REKOM staff members and list the persons without whose active participation it would be just impossible to perform the experiments: A. G. Volkovich, S. V. Smirnov, A. A. Gulyaev, A. P. Govorun, P. F. Strashko, V. L. Shevchenko, A. B. Gaverdovsky, and V. N. Bayushkin. We thank A. A. Rukhadze for useful discussion and for the support of these works.

The experiments were carried out at the RECOM (an affiliate company of the I. V. Kurchatov Institute of
Atomic Energy) at the Kurchatov Institute territory since 2002-2004.

\section{References}

[1] L. I. Urutskoev and D. V. Filippov, "Beta-Stability Condition for the Nuclei of Neutral Atoms," Physics-Uspekhi, Vol. 47, No. 12, 2004, pp. 1257-1260.

[2] F. Bosch, T. Faestermann, J. Friese, F. Heine, P. Kienle, E. Wefers, K. Zeitelhack, K. Beckert, B. Franzke, O. Klepper, C. Kozhuharov, G. Menzel, R. Moshammer, F. Nolden, H. Reich, B. Schlitt, M. Steck, T. Stöhlker, T. Winkler and K. Takahashi, "Observation of Bound-State beta-Decay of Fully Ionized ${ }^{187} \mathrm{Re}:{ }^{187} \mathrm{Re}-{ }^{187} \mathrm{Os}$ Cosmochronometry," Physical Review Letters, Vol. 77, No. 26, 1996, pp. 5190-5193.

[3] M. Jung, F. Bosch, K. Beckert, H. Eickhoff, H. Folger, B. Franzke, A. Gruber, P. Kienle, O. Klepper, W. Koenig, C. Kozhuharov, R. Mann, R. Moshammer, F. Nolden, U. Schaaf, G. Soff, P. Spädtke, M. Steck, T. Stöhlker and K. Sümmerer, "First Observation of Bound-State Beta-Decay," Physical Review Letters, Vol. 69, No. 15, 1992, pp. 2164-2167.

[4] D. V. Filippov, "Increase in the Probability of Allowed Electron Beta Decays in a Superstrong Magnetic Field," Physics of Atomic Nuclei, Vol. 70, No. 2, 2007, pp. 258264.

[5] D. V. Filippov, "Decrease in the Probability of Tritium Decay in an External Electric Field," Physics of Atomic Nuclei, Vol. 70, No. 11, 2007, pp. 1840-1845.

[6] D. V. Filippov, "Increase in the Probability of Forbidden Electron Beta Decays in a Superstrong Magnetic Field," Physics of Atomic Nuclei, Vol. 70, No. 12, 2007, pp. 2016 -2024.

[7] K. A. Kouzakov and A. I. Studenikin, "Bound-state BetaDecay of a Neutron in a Strong Magnetic Field," Physical Review C, Vol. 72, No. 1, 2005, p. 15502.

[8] W. G. Chace and H. K. Moore, "Exploding wires," Plenum Press, New York, 1962.

[9] Yu. L. Bakshaev, P. I. Blinov, V. V. Vikhrev, E. M. Gordeev, S. A. Dan'ko, V. D. Korolev, S. F. Medovshchikov, S. L. Nedoseev, E. A. Smirnova, V. I. Tumanov, A. S. Chernenko and A. Yu. Shashkov, "Study of the Plasma in a Preformed Z-pinch Constriction," Plasma Physics Reports, Vol. 27, No. 12, 2001, pp. 1039-1047.

[10] D. V. Filippov, L. I. Urutskoev, G. Lochak and A. A. Rukhadze, "On the Possible Magnetic Mechanism of Shortening the Runaway of RBMK-1000 Reactor at Chernobyl Nuclear Power Plant," in Condensed Matter Nuclear Science, J. P. Biberian, Ed., World Scientific Publishing Co., Singapore, 2006, pp. 838-853.

[11] A. G. Volkovish, A. P. Govorum, A. A. Gulyaev, S. V. Zhukov, V. L. Kuznetsov, A. A. Rukhadze, A. V. Steblevskii and L. L. Urutskoev, "Experimental Observation of the Distortion of The Uranium Isotopic Relationship and Violation of the Thorium-234 Secular Equilibrium Upon Electric Explosion," Annales de la Fondation Louis 
de Broglie, Vol. 30, No. 1, 2005, pp. 63-70.

[12] L. I. Urutskoev, V. I. Liksonov and V. G. Tsinoev, "Observation of Transformation of Chemical Elements during Electric Discharge," Annales de la Fondation Louis de Broglie, Vol. 27, No. 4, 2002, pp. 701-726.

[13] N. G. Ivoilov and L. I. Urutskoev, "The Influence of "Strange" Radiation on Mössbauer Spectrum of Fe57 in Metallic Foils," Annales de la Fondation Louis de Broglie, Vol. 29, No. Hors ser. 3, 2004, pp. 1177-1186.

[14] G. I. Ramendik, "Elemental Mass-Spectrometric Analysis of Solids," Khimiya, Moscow, 1993 [in Russian].

[15] D. V. Filippov and L. I. Urutskoev, "On the Possibility of Nuclear Transformation in Low-Temperature Plasma from the Viewpoint of Conservation Laws," Annales de la Fondation Louis de Broglie, Vol. 29, No. Hors ser. 3, 2004, pp. $1187-1206$.

[16] V. D. Kuznetsov, G. V. Mishinsky, F. M. Penkov, V. I. Arbuzov and V. I. Zhemenik, "Low Energy Transmutation of Atomic Nuclei of Chemical Elements," Annales de la Fondation Louis de Broglie, Vol. 28, No. 2, 2003, pp. 173- 214.

[17] D. Priem, G. Racineux, G. Lochak, C. Daviau, D. Fargue, M. Karatchentzeff and H. Lehn, "Explosion électrique d'un fil de titane dans de l'eau en milieu confiné," Annales de la Fondation Louis de Broglie, Vol. 33, No. 1-2, 2008, pp. 129-138.

[18] V. A. Erma, "Electron Effects on Barrier Penetration," Physical Review, Vol. 105, No. 6, 1957, pp. 1784-1787.

[19] B. B. Kadomtsev and V. S. Kudryavtsev, "Atoms in a Superstrong Magnetic Field," JETP Letters, Vol. 13, No. 1, 1971, pp. 42-44.

[20] R. P. Taleyarkhan, C. D. West, J. S. Cho, R. T. Lahey Jr., R. I. Nigmatulin and R. C. Block, "Evidence for Nuclear Emissions During Acoustic Cavitation," Science, Vol. 295, No. 5561, 2002, pp. 1868-1873.

[21] F. Cardone, R. Mignani and A. Petrucci, "Piezonuclear Decay of Thorium," Physics Letters A, Vol. 373, No. 22, 2009, pp. 1956-1958. 